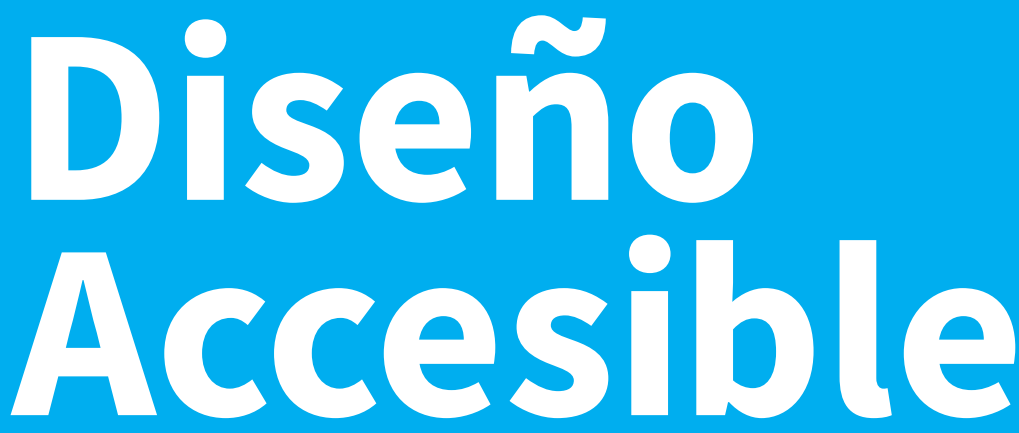

\title{
Diseño Accesible
}

\section{Resumen}

Luego de identificar la población en

Colombia que está en condición de

discapacidad y entender la ausencia de propuestas académicas universitarias en el tema puntual de la accesibilidad, de conocer las normas y obligaciones del Estado para la población discapacitada, se formula las necesidades para crear un programa universitario en diseño accesible que brinde una nueva visión del diseño a todos aquellos que están involucrados en dicho proceso.

\section{Abstract}

After identifying the population in Colombia that is disabled and understanding the absence of university academic proposals

\section{Alfonso Llerena}

\section{Polo}

Consultor en experiencia de usuario, profesional en diseño gráfico y medios audiovisuales, especialista en comunicación multimedia y Magíster en tecnologías accesibles. Diseñadory evaluador de productos, servicios y tecnologías. Docente universitario con experiencia en liderar proyectos de comunicación visual en productos digitales. 
on the specific issue of accessibility, knowing the rules and obligations of the State for the disabled population, the needs to create an academic program on accessible design are formulated that gives a new vision of design to all those who are involved in this parricular process.

\section{Introducción}

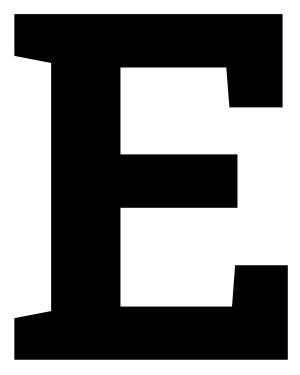

En Colombia, según el Departamento Nacional de Estadística DANE, el número de personas discapacitadas es aproximadamente de dos millones dieciocho mil (2'018.000). Para las instituciones de Educación Superior este valor permite pensar en programas universitarios que en su contenido sean lo suficientemente innovadores y que involucren el concepto de accesibilidad a

Palabras clave sus estudiantes. En el caso de programas en Accesibilidad, Diseño, Accesible,

Discapacidad, Producto de Apoyo,

Educación en Colombia. diseño, es difícil encontrar contenidos que puedan cambiar la ideología tradicional de dichos programas, lo que lo convierte en una alternativa para el desarrollo de nuevas propuestas académicas.

A raíz de la anterior reflexión surge la necesidad de pensar en el diseño del futuro.

Key words Accessibility, Design, Accessible, Disability, Viendo el diseño como un escenario que permite la generación de nuevas propuestas desde lo proyectual, desarrollando un amplio sentido del análisis y su relación con el contexto de uso sobre la condición 
exploratoria innata del ser humano, esta permite la construcción de

diferentes escenarios de investigación proyectual. (Dewey, 1938). Asimismo, en la época actual, la sociedad ubica a la persona por delante de su discapacidad, la accesibilidad se convierte en esa entidad que puede darle al sujeto la posibilidad de ser incluido o excluirlo dentro de su contexto social. Pero es este contexto el que debe dar las condiciones de vida de la persona con discapacidad para que no exista ninguna barrera en su contra. (Bank-Mikkelsen, 1975; Nirje, 1969; Wolfensberger, 1972). En este sentido, estos espacios son, entre otros, los que afectan directamente el campo del diseño. Lo cual permite crear cuestionamientos sobre los contenidos de los programas universitarios para que formen personas consientes de las necesidades y exigencias de las poblaciones más vulnerables, particularmente las personas con algún tipo de discapacidad física. Así, en esta investigación se busca dar respuesta a cuáles son los mecanismos teóricos, pedagógicos, legales y académicos necesarios para crear un programa de Diseño Accesible en Colombia que permita a los participantes aprender, conceptualizar y crear artefactos de diseño basados en la accesibilidad, usabilidad y principios del diseño universal.

\section{Metodología}

Para el desarrollo de este artículo se planteó trabajar en cuatro etapas descritas de la siguiente manera: La primera consistió en identificar y caracterizar el problema; posteriormente se realizó un trabajo de campo en temáticas de accesibilidad en temas como la discapacidad en Colombia, el diseño y las tecnologías de apoyo; al final se analizaron los datos obtenidos y se planteó una propuesta para la creación de un programa académico en Diseño Accesible.

En cuanto a la etapa de identificación, se hizo un estudio que permitió determinar las características del problema, y, luego, se hizo un barrido de los estudios realizados en el país sobre el 
diseño y la accesibilidad. Adicionalmente se buscó información sobre los programas académicos que involucren el tema de la accesibilidad en el país, Latinoamérica y Europa. Al respecto, se obtuvo información valiosa sobre las normativas y leyes existentes que rigen la accesibilidad con el fin de procesarla, analizarla y tener una visión general sobre este aspecto.

En la etapa de indagación, se realizaron encuestas que dieron a conocer la percepción de las personas respecto al programa en Diseño Accesible. En el desarrollo de las encuestas se preguntó a estudiantes, egresados y al personal administrativo de una universidad, cuyo apoyo fue fundamental en esta investigación. Luego se analizó la información, se interpretaron los datos con el fin de dar respuesta al problema planteado en la etapa inicial. Para finalizar se construyó una propuesta para la Creación de un programa de Diseño Accesible en la Universidad Politécnico Grancolombiano (IUPG.) y se hicieron los diferentes trámites legales en Colombia para que el Ministerio de Educación Nacional apruebe el programa.

\section{Resultados}

Luego de observar el estado del arte relacionado con el diseño y la accesibilidad en Colombia, donde se percibe un ambiente favorable en el ámbito internacional y nacional, se observa de manera contundente la falta de ofertas académicas que tienen como base la accesibilidad. Para ello, se realizaron indagaciones que permitieron entender el significado de esta situación. Entre estas, se analizaron factores como la falta de sensibilidad, la falta de conocimientos técnicos y jurídicos que muestren a las entidades de educación las ventajas de incluir dentro de sus programas académicos la accesibilidad como una forma de conocimiento donde se exalta la inclusión e igualdad a todas las personas. También se investigó sobre el conocimiento en accesibilidad de las personas del común, se evaluó el nivel de conocimiento que 
tenían acerca de las leyes, las obligaciones del Estado y los conceptos básicos sobre discapacidad. Esto con el fin de proponer soluciones sobre los resultados obtenidos.

Posteriormente se realizó un estudio sobre las preferencias de los estudiantes al momento de buscar programas de diseño con el objetivo de conocer sus intenciones, limitaciones o posibles temas de interés al respecto. Al finalizar se indagó sobre las instancias que se deben seguir sobre la solicitud de aprobación de un programa académico ante el Ministerio de Educación Nacional en Colombia.

La muestra de personas es interesante ya que confluyen en un mismo espacio tanto físico como digital durante todo el año (directivos, profesores, estudiantes, personal de servicios-aseo, jardinería, vigilancia etc.- y visitantes temporales o de paso). En cada uno de estos conjuntos existen individuos con diversas condiciones de discapacidad. Algunas de ellas no son tan notorias a simple vista pero se destacan problemáticas tales como: Problemas de visión, población adulta, personas con pocas destrezas en el manejo de tecnologías, problemas de movilidad y población con dificultades para acceder a dispositivos tecnológicos.

\section{Caracterización de la Población}

Las características de la población seleccionada para la muestra cumplió con el siguiente perfil: personas de ambos sexos, con edades entre los 18 y 60 años, con problemas de accesibilidad comprobados en baja visión o ciegos. Así mismo, se tuvo en cuenta particularmente personas que utilizaran productos de apoyo de manera periódica. Por otro lado, se recurrió a la población que no tiene discapacidad alguna como complemento al proceso.

- $\quad$ Mayores de 55 años: 20 personas

- Jóvenes entre los 22 y 30 años: 30 personas 
- Personas con Baja visión: 5 personas

- Usuarios de Producto de apoyo: 2 personas

- Sin discapacidad: 40 personas

- Total: 97 personas

\section{Conclusiones}

Es difícil dar conclusiones a un trabajo que se ve como la punta de un Iceberg, advirtiendo que existen tantos tópicos que abarcar, al final el resultado solo es un paso en el largo camino de la accesibilidad en Colombia. Para responder al interrogante: ¿Cuáles son los requisitos teóricos, pedagógicos, legales y académicos necesarios para crear y consolidar un programa de postgrado en Diseño y Accesibilidad en Colombia? Para un programa de pregrado en Diseño Accesible, sería necesario abarcar de manera objetiva los siguientes aspectos analizados en el estado del arte de este documento.

En primer lugar, están los fundamentos sobre discapacidad y accesibilidad, leyes y normas que rigen la discapacidad y accesibilidad a nivel nacional e internacional, la empleabilidad e inclusión laboral, principios de diseño universal y usabilidad de la tecnología. Asimismo, es importante trabajar de la mano con una institución universitaria que tenga la experiencia, trayectoria y reconocimiento a nivel internacional para crear un programa de Diseño Accesible como una propuesta de innovación e inclusión social. Con relación a las cuestiones legales, es necesario regirse por los lineamientos internos de esta universidad y del Ministerio de educación Nacional para proponer el programa, cumplir con los requisitos técnicos y legales para tal fin. Asimismo, es de suma importancia contar con el apoyo del Estado colombiano especialmente con instituciones gubernamentales como el Ministerio de Educación (MEN), Ministerio de tecnologías de la Información 
(MIN TIC) y entes encargados de promover campañas de inclusión académica a personas discapacitadas en el país.

Para responder a la pregunta: ¿Existen iniciativas universitarias donde se tome como base el diseño enfocado en la accesibilidad? Es contundente la respuesta, ya que no existe en Colombia un programa similar. Sí existen algunas iniciativas en áreas diferentes, lo que se trasforma en una ventaja y una posibilidad de innovación para muchas instituciones universitarias.

Si nos cuestionamos sobre los conocimientos que tienen las personas respecto a la accesibilidad, queda un sin sabor que solo da motivos a reflexionar y entender que no podemos seguir siendo pasivos. La discapacidad no debe ser vista con lástima, puesto que todos somos seres en igualdad de condiciones. Los cambios deben iniciar desde la familia, en casa, en la escuela, en la sociedad y en la implementación de políticas. En suma, fue un proceso de reflexión sobre la forma de plantear productos con el menor número de barreras de accesibilidad y ver como se encuentran presentes en el diario vivir.

Para el siguiente interrogante: ¿Qué factores influyen en la decisión de cursar un programa de pregrado en diseño? La sensación es que los jóvenes en Colombia no tienen dentro de su modelo mental la accesibilidad como una alternativa laboral a futuro. Ven el diseño más enfocado hacia áreas como la publicidad, la ingeniería, la comunicación o los medio audiovisuales. La falta de conocimiento, de información no permite que tengan una visión objetiva respecto a su futuro laboral.

A título personal puedo destacar varias conclusiones respecto a este trabajo. En primer lugar, veo con buenos ojos la creación de nuevas opciones de estudio al proponer una nueva especialización con el tema de la accesibilidad como eje central de conocimiento. 
Es una satisfacción personal tener la posibilidad de ayudar a una sociedad que parece ser inmune y poco sensible al tema de la inclusión y reconocimiento de las personas.

Asimismo, considero que en este país no es suficiente hablar de cifras como dos millones de habitantes con problemas de discapacidad. Debemos hablar de 50 millones de habitantes que pueden ser conscientes de aquellos que padecen algún tipo de discapacidad y que están siendo ignorados por el Estado, que el gobierno a pesar de promulgar leyes no ha sido lo suficientemente firme en sus obligaciones. Es decir, que no es suficiente una legislación estricta, los cambios deben ser estructurales, para llegar al ideal de un país accesible. La educación es, entonces, una de las herramientas más poderosas en el cambio de la sociedad. Iniciativas como esta fomentan la difusión y el conocimiento en un tema que por ahora tiene mucho para dar.

\section{Referencias bibliográficas}

Bank-Mikkelsen, N. (1975). El principio de normalización. Revista Siglo Cero, (37), 16-21.

Dewey, J. (1938). Experiencia y educación. Nueva York: Kappa Delta.

Nirje, B. (1969). The normalization principle: implications on normalization. En Symposium on normalization. Madrid: SIIS. En http://usuarios.discapnet.es/disweb2000/ Portadas/24may2004.htm

Wolfensberger, W. (1972). The principle of normalization in human services. Toronto: National Institute on Mental Retardation. 